\title{
Study on Tensile Properties of Nanoreinforced Epoxy Polymer: Macroscopic Experiments and Nanoscale FEM Simulation Prediction
}

\author{
Zhenqing Wang, ${ }^{1}$ Fang Liu, ${ }^{1}$ Wenyan Liang, ${ }^{1}$ and Limin Zhou ${ }^{2}$ \\ ${ }^{1}$ Smart Structures and Advanced Composites Laboratory, College of Aerospace and Civil Engineering, \\ Harbin Engineering University, Harbin 150001, China \\ ${ }^{2}$ Department of Mechanical Engineering, The Hong Kong Polytechnic University, Hong Kong
}

Correspondence should be addressed to Fang Liu; liufang_winter@163.com

Received 15 February 2013; Revised 20 April 2013; Accepted 20 April 2013

Academic Editor: Rui Huang

Copyright (c) 2013 Zhenqing Wang et al. This is an open access article distributed under the Creative Commons Attribution License, which permits unrestricted use, distribution, and reproduction in any medium, provided the original work is properly cited.

The effect of nanosilica contents on mechanical properties of the epoxy matrix with some nanoparticle aggregations was studied in macroscopic experiments and nanoscale simulation, particularly with regard to the effective modulus and ultimate stress. Three analytical models were used to obtain the effective elastic modulus of nanoparticle-reinforced composites. Based on Monte-Carlo method, the special program for the automatic generation of 2D random distribution particles without overlapping was developed for nanocomposite modeling. Weight fractions of nanoparticles were converted to volume fractions, in order to coordinate the content unit in the simulation. In numerical analysis, the weak interface strengthening and toughening mechanism was adopted. Virtual crack closure technique (VCCT) and extended finite element method (XFEM) were used to simulate phenomena of nanoparticle debonding and matrix crack growth. Experimental and simulation results show a good agreement with each other. By way of simulation, the weak interface toughening and strengthening mechanism of nanocomposites is confirmed.

\section{Introduction}

Epoxy resin has been widely used in the aerospace field, as fiber-reinforced composite matrix and adhesive. Cured epoxy resin is a highly cross-linked polymer. However, the structure of thermosetting resin will lead to brittleness of the material, which determines the weak resistance to the fatigue loading.

To toughen epoxy resin, a common method is introducing particles in the resin, including liquid rubbers, thermoplastics, copolymers, silica nanoparticles, silicate layers, core shell particles, and combinations of these. The major toughening mechanisms involve rubber particle debonding/cavitation, localized shear banding of matrix as well as rubber particle bridging. Fracture toughness of rubber toughened epoxy resin will be improved obviously, while many other desirable properties, such as elastic modulus and failure strength, will decrease significantly [1]. In comparison, rigid nanofillers can improve the fracture toughness, stiffness, and even strength of epoxy resin [2-6].
Siddiqui et al. [7] added carbon nanotubes (CNTs) with the weight fraction ranging from $0 \mathrm{wt} . \%$ to $1 \mathrm{wt} . \%$ into epoxy matrix to form nanoparticulate-reinforced composites and found that tensile strength increased gradually, reaching the peak at $0.5 \mathrm{wt} . \%$. Jingchao et al. [8] reported that adding silica nanoparticles in an epoxy had a significant effect on tensile properties of the modified epoxies. When it came to $3 \mathrm{wt} . \%$ of nanosilica, tensile strength reached the maximum. Huang et al. [9] indicated that tensile strength, elongation at break, and impact strength of $\mathrm{SiO}_{2}$ /epoxy nanocomposites increased with the silica weight fraction at first, peaking at $2 \mathrm{wt} . \%$. Bakar et al. [10] found that the addition of $2 \%$ montmorillonite or $5 \%$ polyamide resulted in the best improvement of the impact strength and the critical stress intensity factor relative to the unmodified epoxy resin. Carballeira and Haupert [11] obtained a fluctuant effect of the titanium dioxide content on tensile strength of nanocomposites.

Actually, the distribution of nanoparticles in matrix may be not so perfect. There will be some aggregations (see 


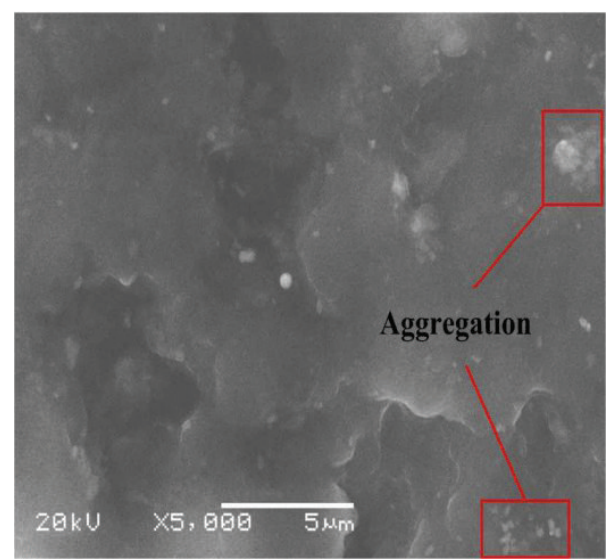

FIGURE 1: Aggregations and uneven distribution of nanoparticles in matrix.

Figure 1). In this work, this factor was taken into account in modeling of simulation.

Recently, it is manifest that, the filler debonding and the subsequent void growth as well as the matrix shear band likely play the key roles in strengthening and toughening [12-17]. In the present work, virtual crack closure technique (VCCT) and extended finite element method (XFEM) were used to simulate phenomena of nanoparticle debonding and matrix crack growth. At the same time, a comparison of numerical and experimental data was carried out.

\section{Experimental}

2.1. Materials. Bisphenol A epoxy resin, E51 (epoxide equivalent weight: $188 \mathrm{~g} / \mathrm{eq}$, and viscosity at $25^{\circ} \mathrm{C}$ : $8000 \sim$ $14000 \mathrm{mPa} \cdot \mathrm{s})$, was used in this experiment. The average particle radius of spherical silica nanoparticles was $25 \mathrm{~nm}$, with a specific surface area of $380 \mathrm{~m}^{2} / \mathrm{g}$. Curing agent and accelerant adopted were phthalic anhydride and triethanolamine, respectively.

The silica nanoparticles were first mixed with the epoxy by means of a mechanical mixer, stirring at room temperature for $6 \mathrm{~h}$, digital heating magnetic mixer at $80^{\circ} \mathrm{C}$ for $4 \mathrm{~h}$, and ultrasonic cell crusher (JY98-IIIDN, $19.5-20.5 \mathrm{KHz}$ and $1200 \mathrm{~W}$ ) for $3 \mathrm{~h}$. After that, the hardener (phthalic anhydride) and accelerant (triethanolamine) were added to the mixtures in a ratio of $100: 50$ and $100: 3$ by weight, respectively, while stirring slowly. The mixtures were then degassed in a vacuum oven (about $-100 \mathrm{KPa}$ ) for about $30 \mathrm{~min}$. Upon the completion of degassing, the vacuum was released and the liquid mixtures were heated, then cast into preheated specimen molds, and cured at $135^{\circ} \mathrm{C}$ for $3 \mathrm{~h}$. Once the specimens were cooled and removed from the molds, they were milled using a surface grinder on both top and bottom surfaces to ensure flatness of specimens. As a baseline for comparison, specimens were also prepared for the cured neat epoxy.

2.2. Samples Preparation. Dumbbell tensile samples were made using a steel mold and both sides were polished by emery paper until all visible marks disappeared. Tensile tests
TABLE 1: The tensile properties of epoxy filled with silica nanoparticles for experiments.

\begin{tabular}{lccccc}
\hline Nanosilica content & 0 wt.\% & 1 wt.\% & 3 wt.\% & 5 wt.\% & 7 wt.\% \\
\hline Young's modulus (GPa) & 3.06 & 3.54 & 4.01 & 4.06 & 4.09 \\
Failure stress (MPa) & 27.00 & 27.90 & 34.28 & 48.11 & 30.83 \\
Modulus improvement (\%) & - & 15.67 & 31.05 & 32.68 & 33.66 \\
Failure improvement (\%) & - & 3.33 & 26.96 & 78.19 & 14.19 \\
\hline
\end{tabular}

were performed at a crosshead speed of $1 \mathrm{~mm} / \mathrm{min}$ at room temperature, according to GB/T 1040. The length, width, and thickness of dumbbell-shape tensile specimen are $150 \mathrm{~mm}$, $20 \mathrm{~mm}$, and $4 \mathrm{~mm}$, respectively.

\section{Experimental Results and Discussion}

The tensile properties of epoxies strengthened by silica nanoparticles are comprehensively listed in Table 1, compared with pure epoxy resin. It can be seen from the table that the modulus of the nanocomposite improves with the increase of the nanofiller content, while tensile strength peaks at $5 \mathrm{wt} . \%$. Maximum improvement of modulus is $34 \%$ at $7 \mathrm{wt} . \%$, and failure stress improvement reaches the largest value, $78 \%$ at $5 \mathrm{wt} . \%$. That is to say, optimal content of nanosilica for both maximum stress and modulus in this paper is approximately $5 \mathrm{wt} \%$.

The fracture surface of neat epoxy is smooth, due to its nature of weak resistance to failure. Fracture surfaces of nanocomposites were examined by SEM to investigate the related strengthening mechanism. In case of epoxy/silica binary sample, the zone in Figure 2 shows not only an obviously rough surface, but also some "sea waves like" features. Compared with the known smooth fracture surface of neat epoxy, these rougher surfaces of nanocomposites, which can absorb more energy before tensile failure, may explain the highly improved tensile properties and the toughening and strengthening mechanism of the polymer matrix due to nanoparticles incorporation.

The expression of nanoparticle contents in the experiments is the weight fraction, while analytical formulation prediction and simulation in the following section will need volume fractions. Therefore, in order to facilitate comparison of results, the two particle content expressions should be unified. The conversion process is (1) using electronic balance to obtain the total mass of a tensile specimen; (2) according to the mass fraction of nanoparticles, calculating the total mass of nanofillers in a specimen; (3) then calculating the total particle surface area, according to the specific surface area $\left(\mathrm{m}^{2} / \mathrm{g}\right)$ of $\mathrm{nm} \mathrm{SiO}_{2} ;(4)$ having known radius of the spherical particle, converting the total surface area to the total volume; (5) according to the known size of the specimen, calculating the approximate volume of specimen; (6) dividing the total volume of specimen by the total particle volume, to get the corresponding volume fraction of nanoparticles.

According to this conversion method, we can get corresponding volume fractions of nanoparticles. In this work, 1 wt. $\%$, 3 wt.\%, 5 wt.\%, and 7 wt.\%, corresponded to 


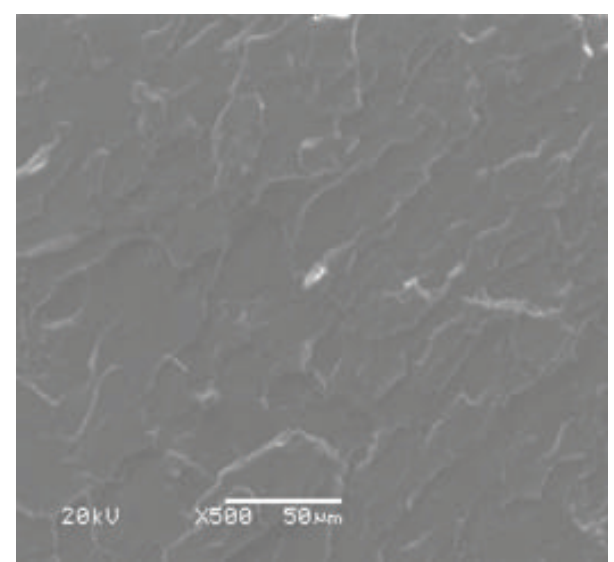

FIGURE 2: SEM graphs of fracture surfaces of tensile samples.

the volume fraction 2.39 vol. $\%, 7.16$ vol. $\%, 11.93$ vol. $\%$, and 16.70 vol. $\%$, respectively.

\section{Prediction and Simulation by FEM}

4.1. Prediction and Comparison with Test. Halpin and Tsai found that the modulus of particulate polymers can be predicted by the semiempirical relationship $[18,19]$ :

$$
\frac{E_{c}}{E_{m}}=\frac{1+A_{1} B_{1} V_{p}}{1-B_{1} V_{p}}
$$

where $A_{1}$ and $B_{1}$ are constants for a given composite. $A_{1}$ is a function of the particle shape and matrix Poisson ratio, and $B_{1}$ is related to the modulus of the particle $\left(E_{p}\right)$ and matrix $\left(E_{m}\right)$.

Guth [20] added a particle interaction term in the Einstein equation which becomes

$$
\frac{E_{c}}{E_{m}}=1+2.5 V_{p}+14.1 V_{p}^{2}
$$

where the linear term is the stiffening effect of individual particles, and the second power term is the contribution of particle interaction.

Counto [21] proposed a simple model for a two-phase particulate composite by assuming perfect bonding between filler and matrix. The composite modulus is given by

$$
\frac{1}{E_{c}}=\frac{\left(1-\sqrt{V_{p}}\right)}{E_{m}}+\frac{1}{\left[\left(1-\sqrt{V_{p}}\right) / \sqrt{V_{p}} E_{m}+E_{p}\right]} .
$$

From comparison of effective modulus from analytical models with experimental results, as shown in Figure 3, it can be seen that, within the content of 12 vol.\%, test results are in good agreement with these analytical formulation prediction, especially for Counto model. While the content is higher, there is a small amount of errors, which may be caused by some aggregations of nanoparticles, due to the high volume fraction.

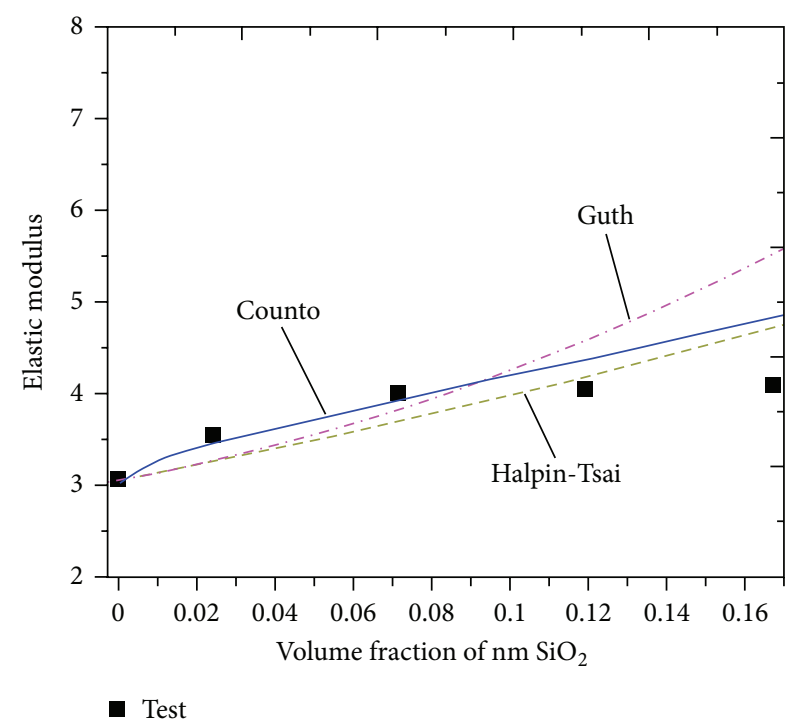

FIGURE 3: Comparison of effective modulus from analytical models with test.

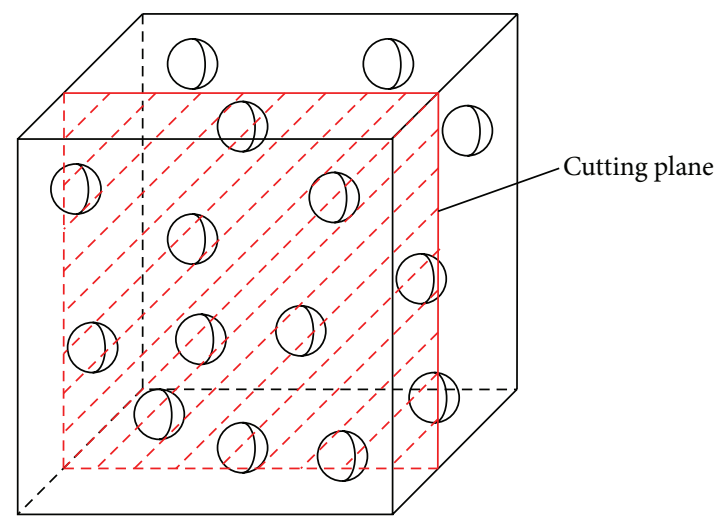

FIGURE 4: Nanoparticles and the cutting plane inside 3D model.

4.2. Modeling Programming. Considering that nanoparticles cannot be uniformly distributed in the matrix completely, there will exist aggregation phenomena. Therefore, random location of nanoparticles should be taken into account in the simulation. As this is a two-dimensional analysis here, the distribution of nanoparticles can be selected from any cutting plane of the 3D model. As shown in Figure 4, there are numerous cutting planes in the three-dimensional model. Any cutting surface will intersect with particles inside 3D model. Since the position of the cutting surface is variable, and thus circle sizes of these particles intersected with the cutting plane will be different. Therefore, considering these situations above, nanoparticle sizes in 2D model in this study are randomly ranging from zero to the whole particle size. Because it is a random distribution problem, the location of the particles in the $2 \mathrm{D}$ model is also random.

In present work, python language is used for modeling programming to simulate the automatic generation of random distribution of nanoparticles in ABAQUS CAE Environment on the basis of Monte-Carlo method. Figure 5 


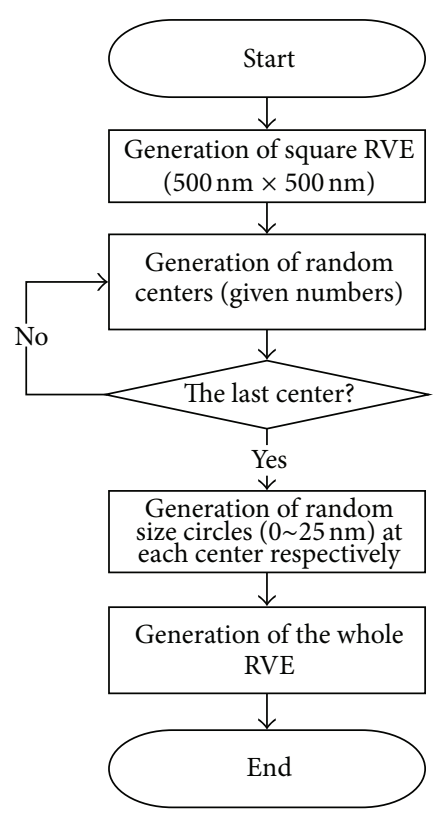

FIGURE 5: Programming process for generation of RVE.

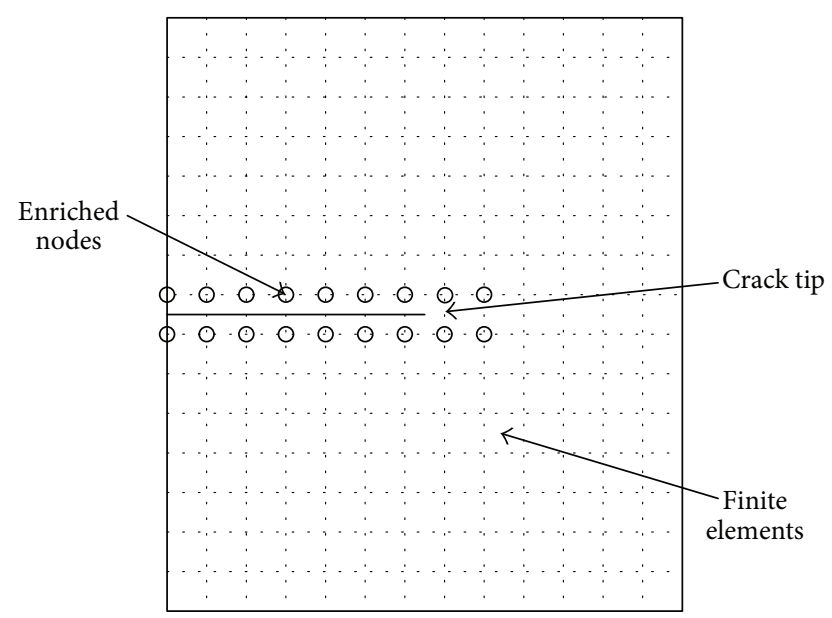

FIGURE 6: One enriching strategy near the crack.

shows programming for generation of representative volume element square (RVE).

In order to generate a random particle distribution RVE, a regular RVE should be presented firstly in this algorithm. Corresponding parameters such as $S, V_{p}$, and $R$ ( $S$ is the side length of RVE. $V_{p}$ is the volume fraction of particles and $R$ is the radius of a particle with the assumption of each particle having a circle section cross) and should be inputted before running the program. Secondly, centers of nanoparticle circles with random coordinate values inside RVE will be generated one by one until it comes to the last center, which is determined by the volume fraction of particles. After all centers are generated automatically, particle circles will be generated subsequently at the corresponding center with random sizes ranging from zero to the whole particle

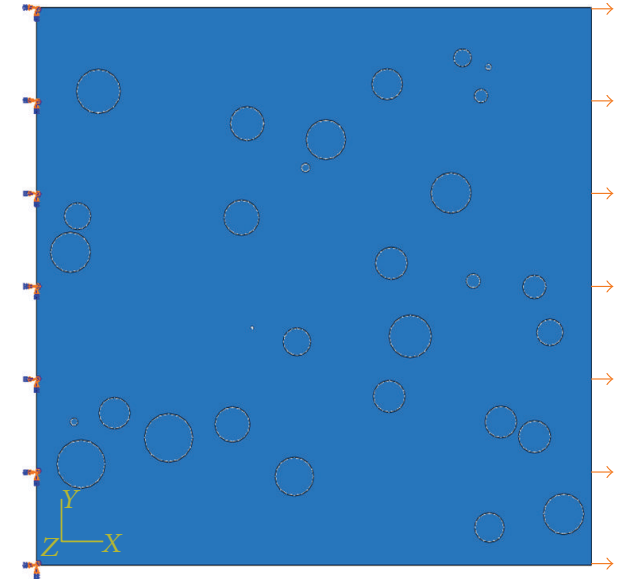

Figure 7: RVE displacement-loading schematic.

size. A random particle distribution RVE sample is shown in Figure 7.

There are some restrictions on particles center generation to avoid overlapping: (a) the minimum distance between particle centers with each other is the particle diameter (50 nm). (b) the minimum distance between particle centers and RVE boundaries is the particle radius $(25 \mathrm{~nm})$.

4.3. Tensile Simulation. The virtual crack closure technique (VCCT, Abaqus Analysis User's Manual 11.4.3) criterion is based on linear elastic fracture mechanics theory, so it can be applied to solve the problem of brittle crack propagation on predefined surfaces, to simulate the interfacial debonding.

In VCCT analysis, node combination technique is used to simulate crack propagation. When the crack propagation criteria, (4), are met, the node in the same location will be separated into two nodes, and the coupled degrees of freedom can be released:

$$
\frac{G}{G_{C}} \geq 1,
$$

where $G$ is the equivalent strain energy release rate and $G_{C}$ is the critical strain energy release rate.

In this paper, simulation will focus on the weak interface toughening mechanism. As a result, interface debonding will occur, followed by crack failure.

The extended finite element method (XFEM, Abaqus Analysis User's Manual 10.6.1) is an effective method for discontinuous problems in mechanics whereby cracks and crack growth can be modeled by finite elements with no remeshing. By this method, a crack arbitrarily aligned within the mesh can be represented by means of enrichment functions, as shown in (5) and Figure 6. The first term on the right-hand side of (5) is the classical finite element (CFEM) approximation to determine the displacement field, while the second term is the enrichment function which takes into account the existence of any discontinuities such as a crack.

To model the crack, basic XFEM approximation is that considering $x$, a point in a finite element model. Also assuming there is a discontinuity in the arbitrary domain 

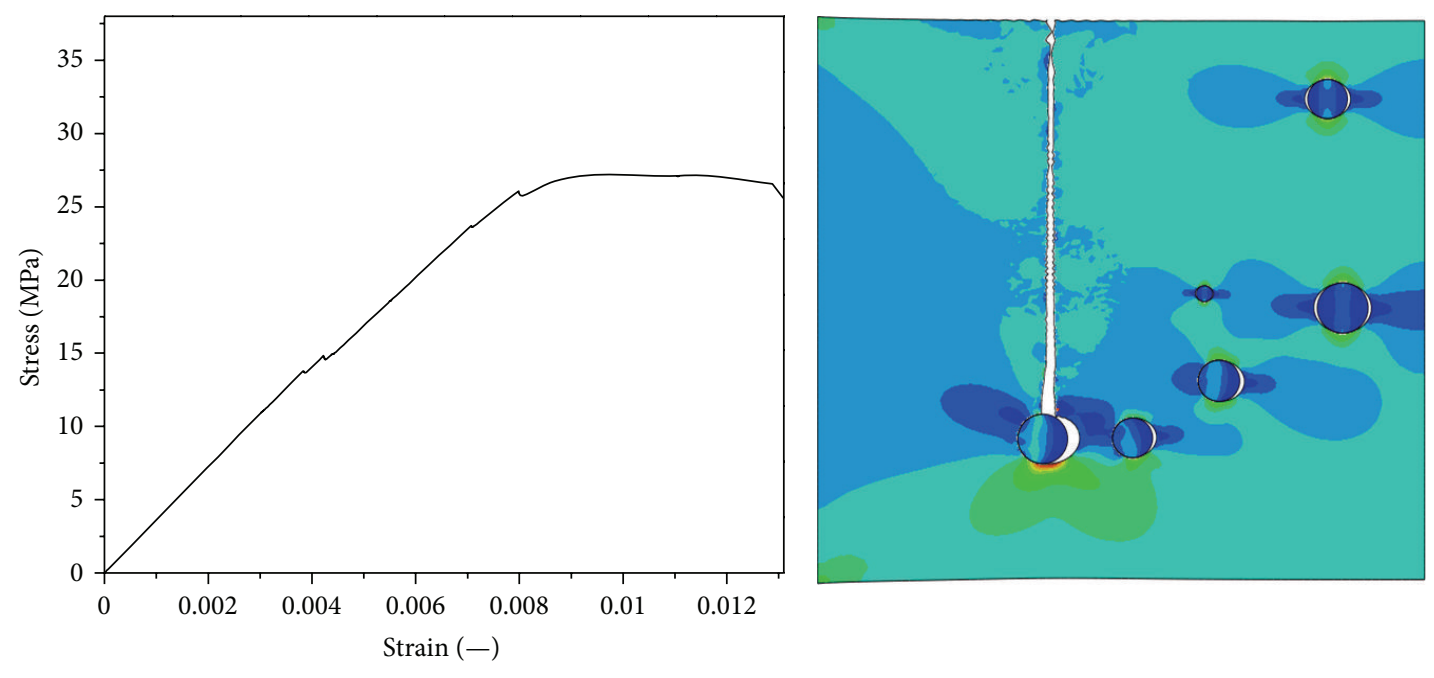

— 1 wt.\% (2.39vol.\%)

(a)
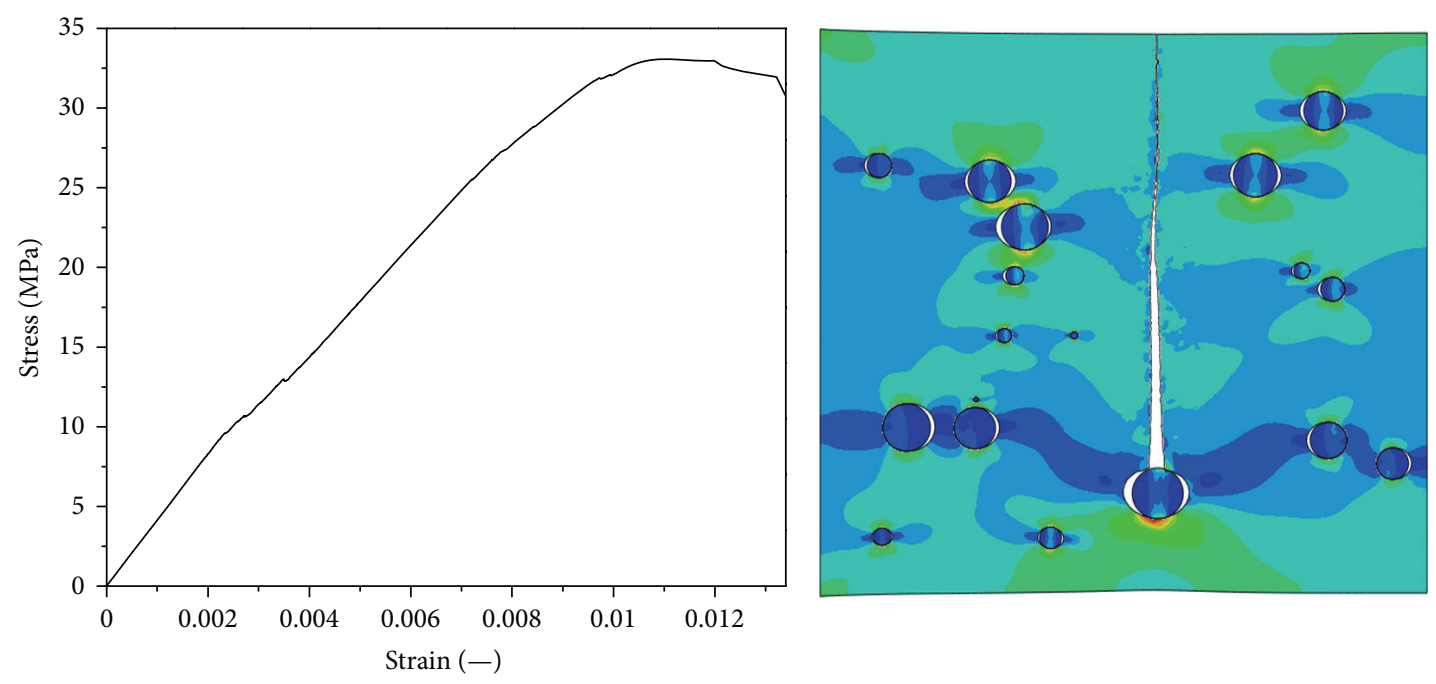

- 3 wt.\% (7.16 vol.\%)

(b)
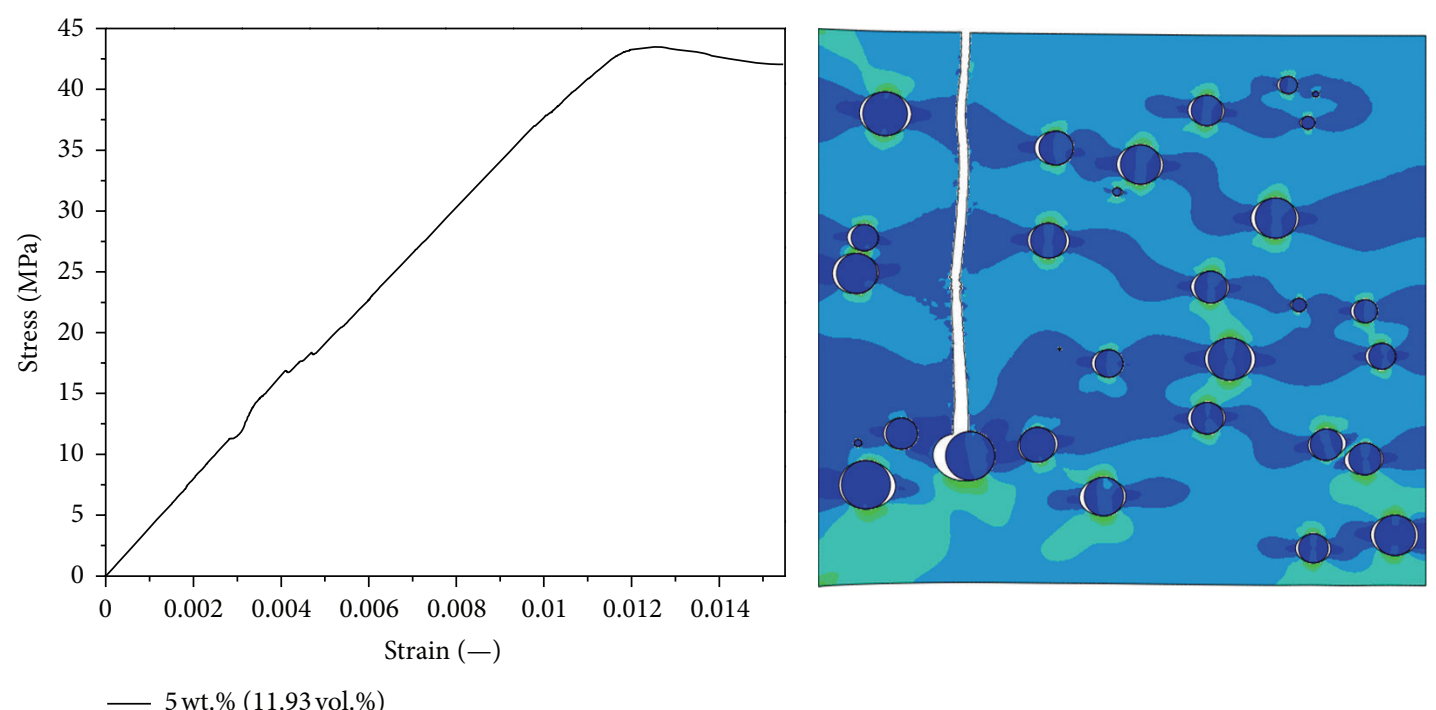

(c)

Figure 8: Continued. 

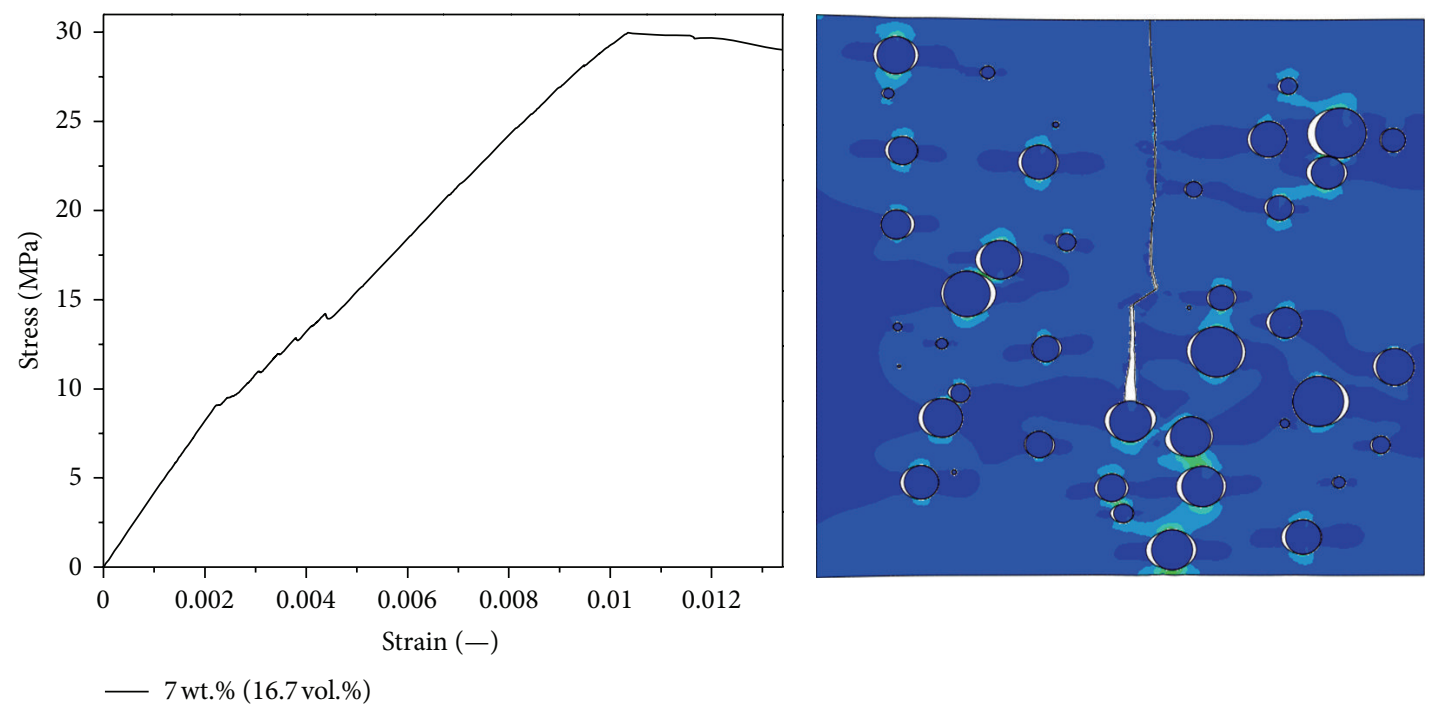

(d)

FIGURE 8: Nanocomposite failure diagrams and corresponding stress-strain curves for different particle contents: (a), (b), (c), and (d).

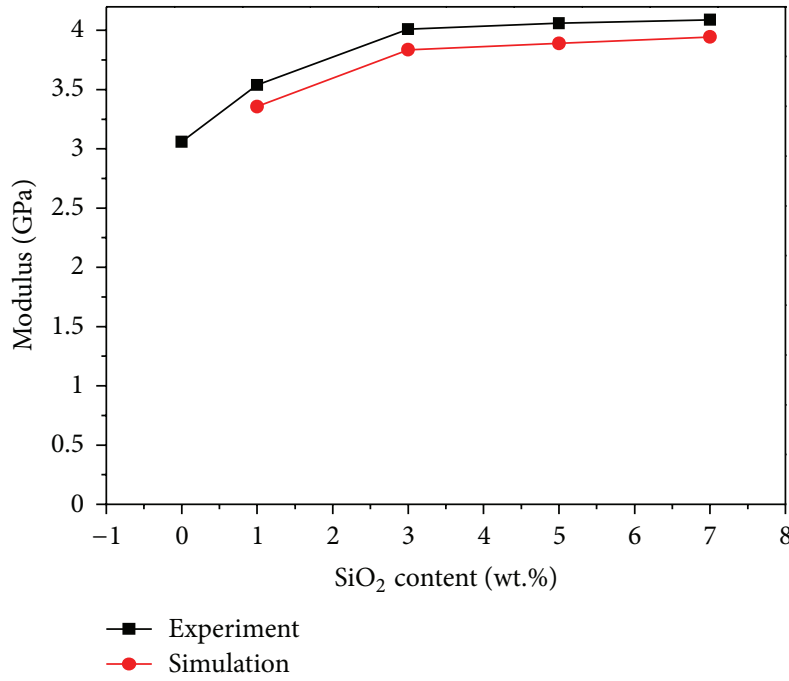

(a)

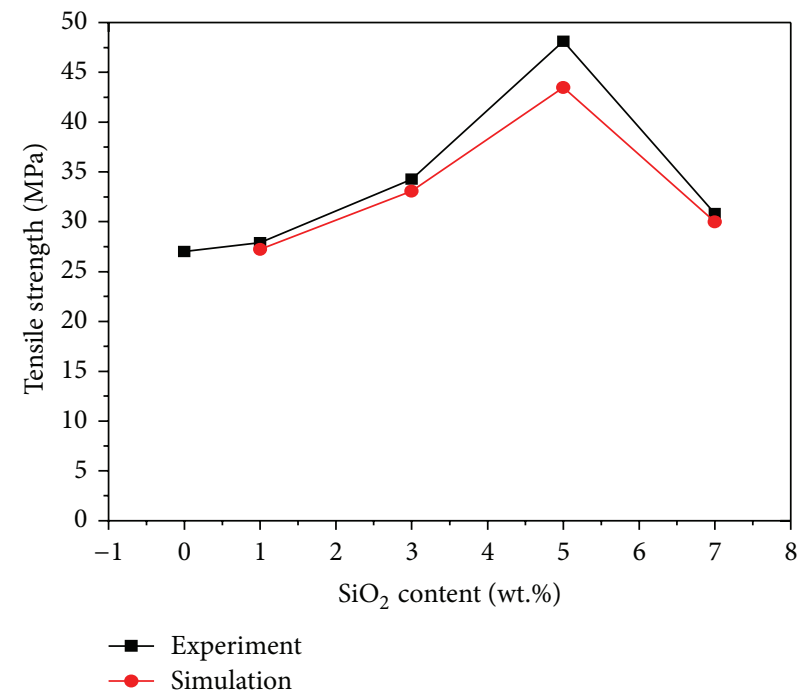

(b)

FIgURE 9: Tensile properties of epoxy resin as a function of nanoparticles $\left(\mathrm{SiO}_{2}\right)$ content.

discretized into some $n$ node finite elements (see Figure 6). In XFEM, the following is utilized to calculate the displacement for the point $x$ locating within the domain [22]:

$$
\mathbf{u}^{h}(\mathbf{x})=\mathbf{u}^{\mathrm{FE}}+\mathbf{u}^{\mathrm{enr}}=\sum_{j=1}^{n} N_{j}(\mathbf{x}) \mathbf{u}_{j}+\sum_{k=1}^{m} N_{k}(\mathbf{x}) \psi(\mathbf{x}) \mathbf{a}_{k},
$$

where $N_{j}(\mathbf{x})$ and $N_{k}(\mathbf{x})$ are finite element shape functions, $\mathbf{u}_{j}$ is the vector of regular degrees of nodal freedom in the finite element method, $\mathbf{a}_{k}$ is the added set of degrees of freedom to the standard finite element model, and $\psi(\mathbf{x})$ is the discontinuous enrichment function defined for the set of nodes that the discontinuity has in its influence (support) domain.
4.4. Simulation Results and Discussion. In this simulation, Young's modulus and failure stress of neat epoxy were $3.06 \mathrm{GPa}$ and $27 \mathrm{MPa}$, respectively, corresponding to parameters obtained in the previous section, while Young's modulus of $70 \mathrm{GPa}$ [23] was adopted for nanosilica. The size of representative volume element square (RVE) selected here for modeling was $500 \mathrm{~nm} \times 500 \mathrm{~nm}$. Figure 7 shows RVE displacement-loading schematic. Tensile analysis in nanoscale was completed by using ABAQUS. Many scholars agree the weak interface toughening and strengthening mechanism, which is adopted in this paper, so the failure process of nanocomposites here is simulated by interface debonding at first and then matrix 
TABLE 2: The average modulus and failure stress values for different nanocomposites in simulation.

\begin{tabular}{lcccc}
\hline Nanosilica content & 1wt.\% & 3 wt.\% & 5 wt.\% & 7 wt.\% \\
\hline Young's modulus (GPa) & 3.356 & 3.837 & 3.890 & 3.943 \\
Failure stress (MPa) & 27.214 & 33.072 & 43.477 & 29.974 \\
\hline
\end{tabular}

failure. Particle debonding and matrix crack failure were achieved by VCCT and XFEM, respectively. Interfaces, with zero thickness, debonded with respect to the energy criterion. Initiation and evolution of matrix failure used the maximum stress criterion and energy criterion, respectively.

The average modulus and failure stress values for different nanocomposites in simulation are shown in Table 2. Figure 8 gives nanocomposite failure diagrams and corresponding stress-strain curves for different particle contents. From stress-strain curves, we can see that there are some fluctuations for stress values during the elastic stage, and the situations of stress fluctuations are diverse. These may be caused by the random nanoparticles of different models and their debonding, and it may be one cause to explain the increasing strength of nanocomposites. Besides, particle debonding and crack growth, which will absorb energy during tensile simulation, may play key roles in toughening and strengthening. For investigating the interaction between nanoparticles and matrix under the tensile load, the nanoscale model is needed in the simulation, while the experimental data comes from the macrospecimen. Cross-scale models may cause a slight difference between macromechanics and micromechanics results, which may be acceptable. Although XFEM gives lower prediction than experiments, it can be seen from Figure 9 that test and simulation results are in good agreement with each other, and they have a similar trend. That is to say, the weak interface toughening and strengthening mechanism of nanocomposites is confirmed by way of simulation, and VCCT and XFEM are effective ways to simulate mechanical behavior of nanocomposites.

\section{Conclusions}

In this paper, mechanical properties of epoxy resins filled with different amounts of rigid silica nanoparticles were investigated. Simulation and test results had a similar trend. That was, with nanofiller content increasing, the tensile strength of nanocomposites increased at first, peaking at some content. After that, there was a declining trend. In addition, the weak interface toughening and strengthening mechanism of nanocomposites was confirmed by simulation (VCCT and XFEM). Therefore, to some extent, predicted results in simulation may reflect macroscopic mechanical properties, and simulation can become an effective method to predict the mechanical property trend of nanocomposites.

The optimal content of nanosilica in this paper was approximately $5 \mathrm{wt} \%$. In the meanwhile, modulus of nanocomposites improved with the increase of the nanofiller content, due to the high modulus ( $70 \mathrm{GPa}$ ) of nanosilica. Within the content of $12 \mathrm{vol} . \%$, test results of nanocomposites for modulus were in good agreement with these analytical formulation prediction especially for Counto model.

\section{Acknowledgments}

The authors gratefully acknowledge the financial support from the China Scholarship Council and the support from fundamental research funds for the central universities (HEUCFZ1003), national natural science foundation of China (no. 11272096), the research fund for the Doctoral Program of Higher Education of China (no. 20112304110015) and fundamental research funds for the central universities (HEUCF130216).

\section{References}

[1] M. Jun, M. S. Mo, X. S. Du, S. R. Dai, and I. Luck, "Study of epoxy toughened by in situ formed rubber nanoparticles," Journal of Applied Polymer Science, vol. 110, no. 1, pp. 304-312, 2008.

[2] J. Ma, M.-S. Mo, X.-S. Du, P. Rosso, K. Friedrich, and H.C. Kuan, "Effect of inorganic nanoparticles on mechanical property, fracture toughness and toughening mechanism of two epoxy systems," Polymer, vol. 49, no. 16, pp. 3510-3523, 2008.

[3] L.-C. Tang, H. Zhang, S. Sprenger, L. Ye, and Z. Zhang, "Fracture mechanisms of epoxy-based ternary composites filled with rigid-soft particles," Composites Science and Technology, vol. 72, pp. $558-565,2012$.

[4] S. Deng, J. Zhang, L. Ye, and J. Wu, “Toughening epoxies with halloysite nanotubes," Polymer, vol. 49, no. 23, pp. 5119-5127, 2008.

[5] S. Zhao, L. S. Schadler, H. Hillborg, and T. Auletta, "Improvements and mechanisms of fracture and fatigue properties of well-dispersed alumina/epoxy nanocomposites," Composites Science and Technology, vol. 68, no. 14, pp. 2976-2982, 2008.

[6] T. H. Hsieh, A. J. Kinloch, K. Masania, J. Sohn Lee, A. C. Taylor, and S. Sprenger, "The toughness of epoxy polymers and fibre composites modified with rubber microparticles and silica nanoparticles," Journal of Materials Science, vol. 45, no. 5, pp. 1193-1210, 2010.

[7] N. A. Siddiqui, E. L. Li, M.-L. Sham et al., "Tensile strength of glass fibres with carbon nanotube-epoxy nanocomposite coating: effects of CNT morphology and dispersion state," Composites A, vol. 41, no. 4, pp. 539-548, 2010.

[8] L. Jingchao, L. Xiaobing, Z. Hualin, Y. Yahui, and F. Wanli, "Study of epoxy Resin Reinf orced and Toughened by $\mathrm{nm} \mathrm{SiO}_{2}$," Chinese Journal of Colloid \& Polymer, vol. 18, no. 4, pp. 15-17, 2000.

[9] C. Huang, Y. Zhang, S. Fu, and L. Li, "Mechanical properties of epoxy composites filled with $\mathrm{SiO}_{2}$ nano particles at room and cryogenic temperatures," Acta Materiae Compositae Sinica, vol. 21, no. 4, pp. 77-81, 2004.

[10] M. Bakar, I. Wojtania, I. Legocka, and J. Gospodarczyk, "Property enhancement of epoxy resins by using a combination of polyamide and montmorillonite," Advances in Polymer Technology, vol. 26, no. 4, pp. 223-231, 2007.

[11] P. Carballeira and F. Haupert, "Toughening effects of titanium dioxide nanoparticles on $\mathrm{TiO}_{2}$ /epoxy resin nanocomposites," Polymer Composites, vol. 31, no. 7, pp. 1241-1246, 2010.

[12] B. Wetzel, P. Rosso, F. Haupert, and K. Friedrich, "Epoxy nanocomposites-fracture and toughening mechanisms," Engineering Fracture Mechanics, vol. 73, no. 16, pp. 2375-2398, 2006. 
[13] J. Liu, H.-J. Sue, Z. J. Thompson et al., "Effect of crosslink density on fracture behavior of model epoxies containing block copolymer nanoparticles," Polymer, vol. 50, no. 19, pp. 46834689, 2009.

[14] S. Zhao, L. S. Schadler, R. Duncan, H. Hillborg, and T. Auletta, "Mechanisms leading to improved mechanical performance in nanoscale alumina filled epoxy," Composites Science and Technology, vol. 68, no. 14, pp. 2965-2975, 2008.

[15] B. B. Johnsen, A. J. Kinloch, R. D. Mohammed, A. C. Taylor, and S. Sprenger, "Toughening mechanisms of nanoparticlemodified epoxy polymers," Polymer, vol. 48, no. 2, pp. 530-541, 2007.

[16] T. Kawaguchi and R. A. Pearson, "The moisture effect on the fatigue crack growth of glass particle and fiber reinforced epoxies with strong and weak bonding conditions part 2 . A microscopic study on toughening mechanism," Composites Science and Technology, vol. 64, no. 13-14, pp. 1991-2007, 2004.

[17] T. Kawaguchi and R. A. Pearson, "The effect of particle-matrix adhesion on the mechanical behaviour of glass filled epoxies. Part 2. A study on fracture toughness," Polymer, vol. 44, pp. 4239-4247, 2004.

[18] J. C. Halpin, "Stiffness and expansion estimates for oriented short fiber composites," Journal of Composite Materials, vol. 3 , pp. 732-734, 1969.

[19] J. C. Halpin and S. W. Tsai, "Effects of environmental factors on composite materials," Tech. Rep. 67-423, 1969, AFML-TR.

[20] E. Guth, "Theory of filler reinforcement," Journal of Applied Physics, vol. 16, pp. 20-25, 1945.

[21] U. J. Counto, "Effect of the elastic modulus, creep and creep recovery of concrete," Magazine of Concrete Research, vol. 16, pp. 129-138, 1964.

[22] T. Belytschko and T. Black, "Elastic crack growth in finite elements with minimal remeshing," International Journal for Numerical Methods in Engineering, vol. 45, no. 5, pp. 601-620, 1999.

[23] K. J. Pascoe, An Introduction to the Properties of Engineering Materials, Van Nostrand Reinhold, London, UK, 3rd edition, 1978. 

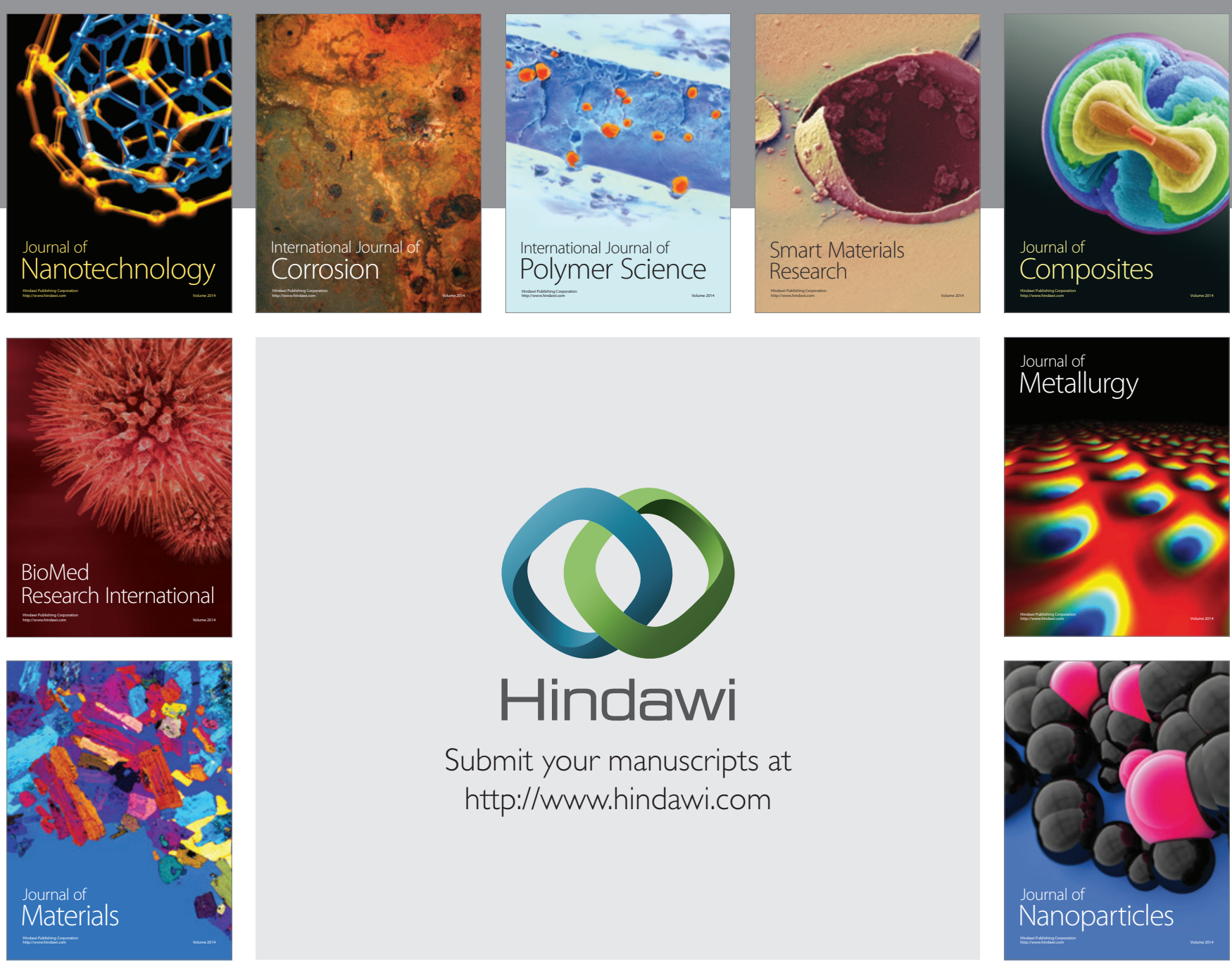

Submit your manuscripts at http://www.hindawi.com
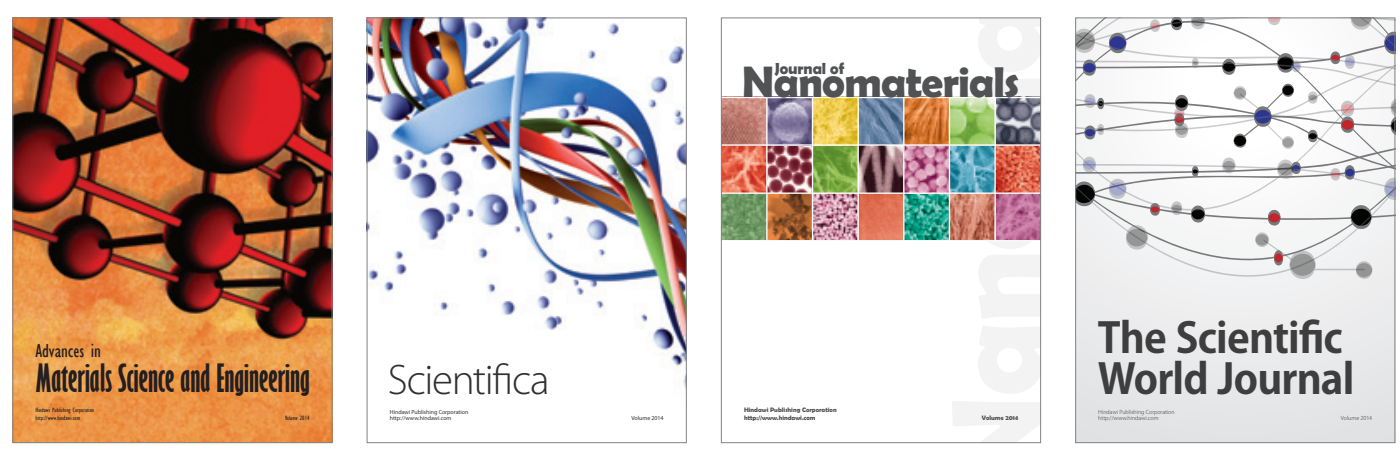

\section{The Scientific World Journal}
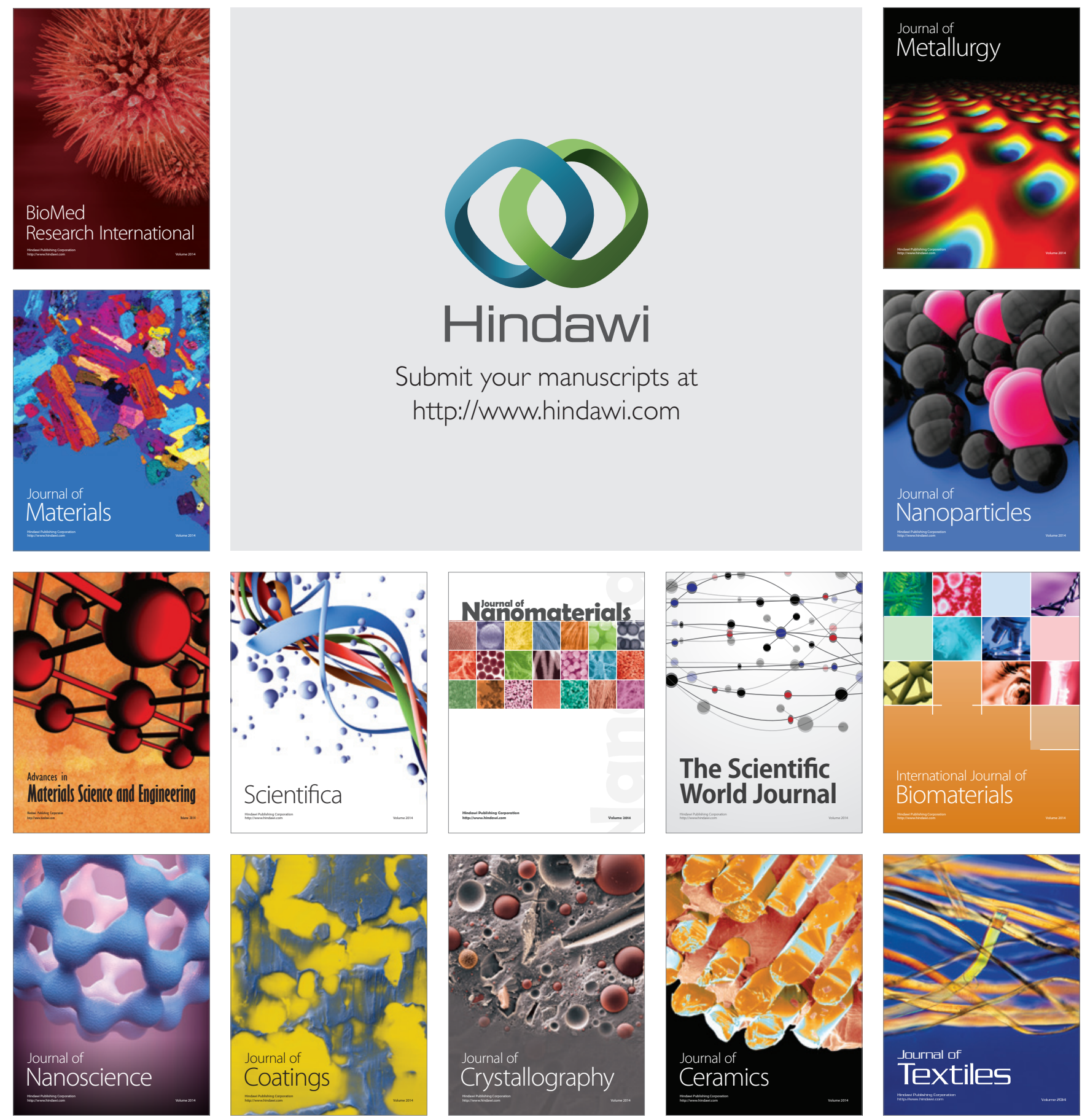